\title{
Key Prediction Security of Keyed Sponges
}

\author{
Bart Mennink \\ Digital Security Group, Radboud University, Nijmegen, The Netherlands \\ b.mennink@cs.ru.nl
}

\begin{abstract}
The keyed sponge is a well-accepted method for message authentication. It processes data at a certain rate by sequential evaluation of an underlying permutation. If the key size $k$ is smaller than the rate, currently known bounds are tight, but if it exceeds the rate, state of the art only dictates security up to $2^{k / 2}$. We take closer inspection at the key prediction security of the sponge and close the remaining gap in the existing security analysis: we confirm key security up to close to $2^{k}$, regardless of the rate. The result impacts all applications of the keyed sponge and duplex that process at a rate smaller than the key size, including the STROBE protocol framework, as well as the related constructions such as HMAC-SHA-3 and the sandwich sponge.
\end{abstract}

Keywords: outer-keyed sponge $\cdot$ full-keyed sponge $\cdot$ key prediction $\cdot$ graph-based proof

\section{Introduction}

Keyed cryptographic functions are desired to "behave like" a random function with the same interface, in such a way that an adversary cannot easily distinguish one from another. Almost all keyed cryptographic schemes have been analyzed in this so-called indistinguishability model, at least from a generic perspective where the underlying primitives are assumed to be sufficiently secure. The indistinguishability model is rather strong: an attack grants an adversary knowledge on some evaluations of the scheme, but not all. It does not automatically produce a full break of the scheme, but rather indicates a non-random property.

Key recoveries are a stronger type of attack. A key recovery can be used to distinguish a scheme from random, but not necessarily the other way around. In some cases, the best distinguishability attack is (close to) a key recovery attack. For example, AES [FIP99] supports keys of size 128, 192, or 256 bits, and the best distinguishing attack on AES known to date is (close to) the generic key recovery attack that succeeds if the attacker makes a total amount of $2^{k}$ offline evaluations of AES, where $k$ is the key size. In a bit more detail, it is generally believed that the strong pseudorandom permutation (SPRP) security of AES is around $t / 2^{k}$, where $t$ is the number of offline evaluations of AES.

It is not always straightforward to achieve security against key recovery attacks up to $2^{k}$. An earlier example of this is the authenticated encryption scheme McOE-X [FFL12], for which Mendel et al. [MMRT12] described a simple key recovery attack in $2^{n / 2}$ evaluations, where $n$ equals both key and state size. Fuhr et al. [FLS15] described sophisticated birthday-type key recovery attacks on CAESAR candidates Marble [Guo14] and AEZ v3 [HKR14]. The designers of AEZ revised their scheme to AEZ v4 in order to mitigate the attack [HKR15a], but subsequently, Chaigneau and Gilbert [CG16] showed that AEZ $\mathrm{v} 4.1$ [HKR15b] is still vulnerable to a key recovery attack with similar complexity. 


\section{$1.1 \quad$ Keyed Sponges}

We will focus on keyed versions of the sponge construction by Bertoni et al. [BDPV07]. The (keyless) sponge construction is a hash function mode that operates on a $b$-bit state, split into an inner part of capacity $c$ and an outer part of rate $r$, where $c+r=b$. Data is absorbed, and the digest is extracted, block-by-block via the outer part of the state, interleaved with evaluations of a $b$-bit permutation $\pi$. The function is proven [BDPV08] to achieve $c / 2$-bit security in the indifferentiability framework [MRH04]. It has been adopted, among others, in the SHA-3 hashing standard [FIP15].

The first, and perhaps simplest, approach of transforming the sponge into a pseudorandom function (PRF) is by Bertoni et al. [BDPV11c], who suggested to simply prepend the message $m$ with the key $K$, i.e., to evaluate the sponge on input $(K \| m)$. The scheme got renewed analysis by Andreeva et al. [ADMV15], who dubbed it the "outer-keyed sponge (OKS)," and later Naito and Yasuda [NY16]. OKS is depicted in Figure 1.

Alternatively, one can design a PRF by just initializing the inner part with the key $K$ instead of with $0^{c}$. This construction, now known as the "inner-keyed sponge (IKS)," was introduced by Chang et al. $\left[\mathrm{CDH}^{+} 12\right]$ and received improved analysis by Andreeva et al. [ADMV15] and Naito and Yasuda [NY16].

Finally, it appeared that secrecy of the state after key injection could be used to support full-state absorption: instead of absorbing data in the $r$-bit outer part only, one could absorb over the entire $b$-bit state. The idea appeared first in the donkeySponge [BDPV12]. An analysis for one output block only was given by Gaži et al. [GPT15a]. A complete security treatment of the "full-keyed sponge (FKS)" was given by Mennink et al. [MRV15] and Daemen et al. [DMV17]. FKS is depicted in Figure 1.

Generically, in terms of the number of permutation evaluations, ${ }^{1}$ the full-keyed sponge is more efficient than the outer-keyed and inner-keyed sponge: data is absorbed $b$ bits at a time rather than $r<b$ bits. From a security perspective, the modes achieve approximately the same level of security. In detail, all achieve a security level of around

$$
\frac{M^{2}}{2^{c}}+\frac{M N}{2^{c}}+\mathbf{A d v}_{F}^{\text {key-pre }}(N)
$$

(omitting constants and details, see Section 4), where $M$ denotes the query complexity to the construction (in terms of the number of underlying primitive calls), $N$ the query complexity to the underlying permutation, and $\mathbf{A d v}_{F}^{\text {key-pre }}(N)$ is the probability that the adversary made queries to the underlying permutation that match the key input to $F \in\{\mathrm{OKS}, \mathrm{IKS}, \mathrm{FKS}\}$. Intuitively, the first two terms of (1) correspond to distinguishing $F$ from a random function, and the last term represents a key prediction, an isolated event in typical security analyses (there is a subtle difference between a key prediction and a key recovery, as we will explain in Section 5).

Note that all sponges achieve a security level of $c$ bits at best, and there is no reason to take a key of size $k>c$. As such, FKS has made IKS obsolete: both require adaptation of the keyless sponge algorithm, both take one evaluation of $\pi$ to absorb the key, both are approximately equally secure, but the FKS is more efficient. FKS does not necessarily make OKS obsolete: although it is less efficient, OKS does not require an adaptation of the keyless sponge algorithm, and can evaluate it in a black-box manner. This happens, for example, in the STROBE protocol framework [Ham17a, Ham17b], as we will elaborate upon in Section 1.4. We restrict our focus to OKS and FKS.

\footnotetext{
${ }^{1}$ The full-keyed sponge would need a permutation with more rounds in order to compensate for the increased adversarial power.
} 


\subsection{Key Prediction Security}

For FKS, using that $k<b$ without loss of generality, it is easy to see that

$$
\operatorname{Adv}_{\mathrm{FKS}}^{\text {key-pre }}(N) \leq N / 2^{k} .
$$

Indeed, the key is absorbed using one evaluation of $\pi$, the adversary can make $N$ attempts, and succeeds if one of those is performed for the corresponding key.

For OKS, the $k$-bit key is partitioned into $r$-bit blocks. If $k \leq r$, then the key prediction term is bounded as before (the key is absorbed using one call to $\pi$ ). On the other hand, if $k>r$, then the key is processed using more than one evaluation of $\pi$, and the current state of the art suggests only $2^{k / 2}$ security. In more detail, Gaži et al. [GPT15b, Lemma 12] (full version of [GPT15a]) derived the following bound for the key prediction term:

$$
\operatorname{Adv}_{\text {OKS }}^{\text {key-pre }}(N) \lesssim\left\{\begin{array}{l}
N / 2^{k}, \text { if } k \leq r \\
b^{\lambda / 2} N / 2^{k / 2}, \text { if } k>r
\end{array}\right.
$$

where $\lambda:=\lceil k / r\rceil$ denotes the number of permutation calls to process the key, and where negligible terms are omitted (refer to Proposition 1 for the details).

This bound shows a counter-intuitive and devastating loss in the key size: whereas one key block still yields the intuitively optimal bound of $N / 2^{k}$ ("one needs to make around $2^{k}$ attempts to recover the key"), once the key size exceeds the rate one loses half the key! The conclusion is that the bound of (3) is, obviously, non-tight.

In Section 3, we derive an improved bound on the key prediction security, and demonstrate that

$$
\operatorname{Adv}_{\mathrm{OKS}}^{\text {key-pre }}(N) \lesssim c^{\lambda-1} N / 2^{k}
$$

where, again, $\lambda:=\lceil k / r\rceil$, and negligible terms are omitted (refer to Theorem 1 for the details). For $k \leq r$ (or $\lambda=1$ ), the earlier bound of Gaži et al. was already tight and the new bound matches it. For $k>r$ (or $\lambda>1$ ), our bound demonstrates that close to $k$-bit security is achieved, with a logarithmic degradation in $c$. This innocent logarithmic loss, in turn, comes from the probability of a lucky multi-collision; it was already present in the bound of Gaži et al., but we slightly improved it. (We remark that the best attack against key prediction has a success probability of around $N / 2^{k}$.)

The result is proven using a graph-based approach, wherein every edge corresponds to an $r$-bit key block guess and one considers the maximum number of paths of length $\lambda$ departing from root node $0^{b}$. We then consider configurations of paths of length $\lambda$, corresponding to the direction in which the $\lambda$ individual queries to the underlying permutation are made. The proof is inspired by that of Gaži et al. [GPT15b, Lemma 12], who also adopted a graph-based approach and introduced the term of configurations, yet it differs in many aspects. Most importantly, Gaži et al. observed that in any configuration, at least half is in the same direction (either forward or inverse). They subsequently use a bad event based on multi-collisions to upper bound the number of possibilities over these $\geq \lambda / 2$ edges, but ignore the problem of the adversary to find the connections over the remaining $\leq \lambda / 2$ edges in reverse direction. This simplification leads to discarding half of the key (hence the denominator $2^{k / 2}$ in (3)). In our bound, we perform an inductive argument on $\lambda$. We prove that for every added layer extension, the size of the yield, or equivalently the number of paths from $0^{b}$, depends only on the presence of multi-collisions and is independent of the configuration of the query.

\subsection{Further Appearances of Key Prediction Security}

The main functionality of the keyed sponge is authentication. It can be used for (authenticated) encryption via the duplex construction by Bertoni et al. [BDPV11a]. The duplex is 
a stateful construction: a call to its initialization interface initializes a state, and a call to its duplexing interface absorbs a data block, applies the permutation $\pi$ on the state, and squeezes at most $r$ bits. Similar to the keyed sponges, the absorption of the data block can be performed over the outer part only ( $r$ bits) or over the entire state $(b$ bits). We will refer to these two as the outer-keyed duplex (OKD) and the full-keyed duplex (FKD). It is important to note that these protocols do not extract data during absorption of the key; in other words, the key is absorbed as if the function is an outer-keyed, resp. full-keyed, sponge.

The security of keyed duplexes can be proven from keyed sponges and vice versa [MRV15, DMV17]. The reduction makes use of the fact that one evaluation of the keyed duplex consists of $\ell$ evaluations of a keyed sponge, where $\ell$ denotes the number of blocks that are duplexed in-between two initialization calls. Even stronger, both the outer-keyed duplex and full-keyed duplex are proven to also achieve the bound of (1), up to constant, where the key prediction security is still for the keyed sponge function $F \in\{\mathrm{OKS}, \mathrm{FKS}\}$.

For both variants of the keyed sponges and keyed duplexes one can also consider security in the nonce-respecting setting, where a nonce is absorbed into the state prior to the first data block. In this case, the general bound of (1) can go down to around

$$
\frac{M^{2}}{2^{b}}+\frac{N}{2^{c}}+\operatorname{Adv}_{F}^{\text {key-pre }}(N),
$$

using techniques of [JLM14, DMV17] (see also Section 4). Common term in the bounds of (1) and (5) is the presence of the term $\mathbf{A d v}_{F}^{\text {key-pre }}(N)$ for $F \in\{\mathrm{OKS}, \mathrm{FKS}\}$. Our improved analysis of $\mathbf{A d v}_{\mathrm{OKS}}^{\text {key-pre }}(N)$ in (4) immediately yields an improved bound for the outer-keyed duplex, as well as for the nonce-respecting variants of both the outer-keyed sponge and duplex.

Beyond the keyed sponges and keyed duplexes, key prediction security also appears in the analyses of HMAC-SHA-3 [NW16] and the sandwich sponge [Nai16]. In these works, the authors adopted the old bound of Gaži et al. of (3). Our new bound of (4) directly improves the security bounds of these schemes.

\subsection{Application}

Despite that FKS generically improves over OKS both from a security as an efficiency perspective, there are still reasons to resort to OKS. First, the security results only focus on the generic construction: full-state absorption allows the adversary more power, and the underlying permutation may likely need more rounds. Second, the usage of OKS is conceptually simpler: the PRF can be implemented using the keyless sponge algorithm as a black-box.

\subsubsection{STROBE}

This exact idea lies at the heart of the STROBE protocol framework [Ham17a,Ham17b]. It is designed on top of the sponge construction, and extends the use of a single permutation to a lightweight framework for network protocols, that allows for (keyless) hashing, authenticated encryption, authentication, pseudorandom number generation, and many more. In order to allow for a simple framework with extremely small code size, all functionalities supported by STROBE operate on the outer part only. In particular, STROBE does not allow for full-state absorption but rather absorbs key and data in the outer part.

The STROBE protocol framework is based on SHAKE [NIS16] and supports instantiations with various widths, as indicated in Table 1. Whereas the larger STROBEs have $k \leq r$, the more lightweight STROBE-128/400 has suggested key size larger than $r$. The same applies to "STROBE lite" on 200-bits state (which is not included in the table). Our analysis confirms that these instantiations do achieve the claimed level of security and 
Table 1: Suggested parameters in the STROBE protocol framework [Ham17a, Ham17b].

\begin{tabular}{lrrrr}
\hline scheme & $b$ & $c$ & $r$ & $k$ \\
\hline STROBE-128/1600 & 1600 & 256 & 1344 & 256 \\
\hline STROBE-256/1600 & 1600 & 512 & 1088 & 256 \\
\hline STROBE-128/800 & 800 & 256 & 544 & 256 \\
\hline STROBE-256/800 & 800 & 512 & 288 & 256 \\
\hline STROBE-128/400 & 400 & 256 & 144 & 256 \\
\hline
\end{tabular}

that the effective key length is not halved (as suggested by the earlier bound of (3)). We remark that the 256-bit key size in the STROBE protocol framework is a mere suggestion by the author; implementers may opt to use a larger or smaller key.

\subsubsection{CAESAR Competition}

Multiple submissions to the CAESAR competition for the development of a portfolio of authenticated encryption schemes [CAE18] adopted the keyed duplex. Focusing on the third round candidates, Table 2 lists the parameters $(b, c, r, k)$ of the four sponge-based schemes Ascon [DEMS16], Ketje [BDP $\left.{ }^{+} 16 \mathrm{a}\right]$, Keyak [BDP $\left.{ }^{+} 16 \mathrm{~b}\right]$, and NORX [AJN16]. Keyak implements the full-keyed duplex construction of [DMV17] and the original key prediction bound of (2) applies. The remaining three schemes evaluate the outer-keyed duplex, with one twist: despite that message is absorbed in the outer part only, the key is absorbed in a full-state fashion. For example, for Ascon-128, the 128-bit key is used (alongside the initialization vector and nonce) to initialize the 320-bit state, and departing from that state, the outer-keyed duplex (with rate $r=64$ ) is evaluated. Therefore, also for these schemes the original key prediction bound of (2) applies. Our observations nevertheless demonstrate that if, for some reason, full-state absorption of the key is infeasible, multi-round outer part absorption does not degrade security.

\subsubsection{Lightweight Permutations}

Our analysis improves over the existing bounds for OKS in case the key size exceeds the rate. It becomes particularly relevant in the context of lightweight cryptography and the current abundance in "small" permutations, all with different features. Whereas most allow for a large enough capacity and rate so that key absorption takes only one round (typically permutations of size $\geq 384$ bits, including Keccak- $f$ [400] [BDPV11b], C-Quark [AHMN10], SPONGENT-256/256/128 [BKL $\left.{ }^{+} 11\right]$, and Gimli $\left.\left[\mathrm{BKL}^{+} 17\right]\right)$, some have a smaller state, such as 228-bit Photon-256/32/32 [GPP11], 320-bit Ascon [DEMS16], 200- or 280-bits PRIMATEs $\left[\mathrm{ABB}^{+} 14\right]$, and 128- or 256-bits Prøst [KLL $\left.{ }^{+} 14\right]$. If the rate is too small and one does not opt to simply initialize the state using the key (as, e.g., described for Ascon above), key absorption is necessarily performed in multiple rounds.

\section{Preliminaries}

For a finite set $\mathcal{S}$, we denote by $s \stackrel{\$}{\leftarrow} \mathcal{S}$ the uniform random sampling of $s$ from $\mathcal{S}$. For a natural number $b \in \mathbb{N},\{0,1\}^{b}$ denotes the set of $b$-bit strings, and perm $(b)$ denotes the set of all permutations $\pi:\{0,1\}^{b} \rightarrow\{0,1\}^{b}$. We denote by $\{0,1\}^{*}$ the set of all strings. For $m \in\{0,1\}^{*}$, we denote by $m_{1}, \ldots, m_{\nu}=s_{b}(m)$ the two-step process of (i) appending $m$ with $10^{-|m|-1 \bmod b}$, and (ii) partitioning the resulting string into $\nu \geq 1$ strings of $b$ bits. 
Table 2: Parameters of the four sponge-based round 3 CAESAR candidates. For Ketje and Keyak, we have taken the recommended key length.

\begin{tabular}{lrrrr}
\hline scheme & $b$ & $c$ & $r$ & $k$ \\
\hline Ascon [DEMS16] & 320 & 256 & 64 & 128 \\
& 320 & 192 & 128 & 128 \\
\hline Ketje [BDP ${ }^{+}$16a] & 200 & 184 & 16 & 92 \\
& 400 & 368 & 32 & 128 \\
\hline Keyak [BDP ${ }^{+}$16b] & 800 & 256 & 544 & $128 . .224$ \\
& 1600 & 256 & 1344 & $128 . .224$ \\
\hline NORX [AJN16] & 512 & 128 & 384 & 128 \\
& 1024 & 256 & 768 & 256 \\
\hline
\end{tabular}

For a $b$-bit string $m \in\{0,1\}^{b}$ and values $c, r \in \mathbb{N}$ such that $c, r \leq b$, we denote by $\lfloor m\rfloor_{c}$ the $c$ rightmost bits of $m$ and by $\lceil m\rceil_{r}$ the $r$ leftmost bits of $m$.

An adversary $\mathcal{A}^{\mathcal{O}}$ is a computationally unbounded algorithm that is given adaptive access to an oracle $\mathcal{O}$ and outputs certain data. It wins if its output fulfills a (possibly randomized) winning condition $\mathrm{W}$. The queries that $\mathcal{A}$ makes are typically recorded in a transcript $\mathcal{Q}$. If $\mathcal{O} \in \operatorname{perm}(b)$, such a transcript $\mathcal{Q}$ consists of a finite amount of tuples $(C, s, t)$, where $s$ is the input to the permutation, $t$ the output of the permutation, and $C \in\{0,1\}$ the query direction ( 0 for forward and 1 for inverse). The query direction $C$ is left implicit if it is irrelevant.

\subsection{General Keyed Sponge}

This work is concerned with the outer-keyed sponge and the full-keyed sponge: the former absorbs data in the outer part only, the latter absorbs data over the entire state. We can therefore neatly describe both in one go by considering a general keyed sponge that operates with two capacities: $c_{\mathrm{ab}}$ for absorption and $c_{\mathrm{sq}}$ for squeezing. (Nevertheless, most of the results in the remainder of the work concern the outer-keyed sponge.)

Let $b, c_{\mathrm{ab}}, c_{\mathrm{sq}}, r_{\mathrm{ab}}, r_{\mathrm{sq}}, k \in \mathbb{N}$ be such that $b=c_{\mathrm{ab}}+r_{\mathrm{ab}}=c_{\mathrm{sq}}+r_{\mathrm{sq}}$. Define $\lambda:=\left\lceil k / r_{\mathrm{ab}}\right\rceil$. The general keyed sponge is a pseudorandom function based on a permutation $\pi \in \operatorname{perm}(b)$ that takes as input a key $K \in\{0,1\}^{k}$, an arbitrary-sized message $M \in\{0,1\}^{*}$, and a natural number $\ell \in \mathbb{N}$, and outputs a value $z \in\{0,1\}^{\ell}$ :

$$
\mathrm{GKS}^{\pi}:(K, m, \ell) \mapsto z \in\{0,1\}^{\ell}
$$

The function is specified in Algorithm 1 and depicted (for integral $\lambda:=k / r_{\mathrm{ab}}$ ) in Figure 1.

The general keyed sponge construction covers the outer-keyed sponge OKS ${ }^{\pi}$ for $\left(c_{\mathrm{ab}}, r_{\mathrm{ab}}\right)=\left(c_{\mathrm{sq}}, r_{\mathrm{sq}}\right)=(c, r)$. It covers the full-keyed sponge $\mathrm{FKS}^{\pi}$ for $\left(c_{\mathrm{ab}}, r_{\mathrm{ab}}\right)=(0, b)$ and $\left(c_{\mathrm{sq}}, r_{\mathrm{sq}}\right)=(c, r)$.

Note that the parameter $\lambda$ indicates the number of invocations of $\pi$ to process the key. As both the outer-keyed as the full-keyed sponge achieve a security level of roughly $c_{\mathrm{sq}}$ at best, we can w.l.o.g. assume that $k \leq c_{\mathrm{sq}}$ throughout. For FKS, this implies that we always have $\lambda=1$. For OKS, $\lambda$ may be larger.

\subsection{Yield and Configurations}

For proper understanding of the security of OKS and FKS against key prediction security as defined in Section 3, we will introduce the yield of a transcript of input-output tuples of $\pi$. 

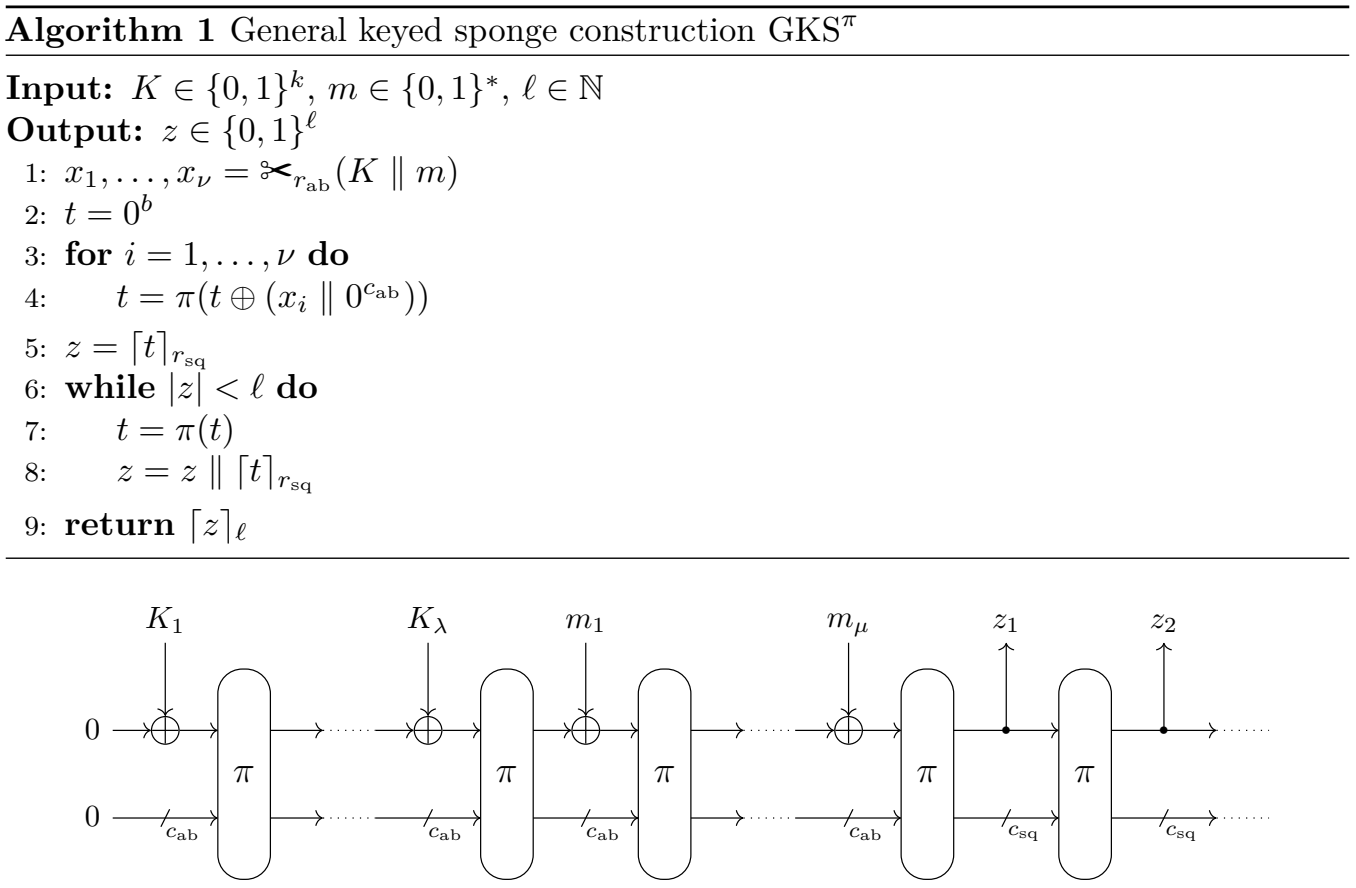

Figure 1: General keyed sponge for $\lambda$ integral key blocks and $\mu$ message blocks. The scheme covers the outer-keyed sponge $(\mathrm{OKS})$ for $c_{\mathrm{ab}}=c_{\mathrm{sq}}=: c$ and the full-keyed sponge $(\mathrm{FKS})$ for $c_{\mathrm{ab}}=0$ and $c_{\mathrm{sq}}=: c$.

Definition 1. Consider GKS based on $\pi \in \operatorname{perm}(b)$, and let $\mathcal{Q}$ be a transcript of $\pi$. The yield yield $\cos _{c_{\mathrm{a}}, \lambda}(\mathcal{Q})$ of $\mathcal{Q}$ is defined as the set of all keys $K \in\{0,1\}^{k}$ for which there exists a message $m \in\{0,1\}^{*}$ such that the first $\lambda=\left\lceil k / r_{\mathrm{ab}}\right\rceil$ evaluations of $\pi$ in line 4 of Algorithm 1 are in $\mathcal{Q}$

In other words, yield $c_{c_{\mathrm{ab}}, \lambda}(\mathcal{Q})$ is the set of keys for which the absorption in GKS can be performed by only reading data from $\mathcal{Q}$, hence, without evaluating $\pi$.

The yield is closely related to a directed acyclic graph $G_{c_{\mathrm{ab}}, \lambda}(\mathcal{Q})=(V, A)$ defined as follows. Vertex $\operatorname{set}^{2} V=\left\{V_{0}, V_{1}, \ldots, V_{\lambda}\right\}$ consists of $\lambda+1$ layers, where

- $V_{0}=\left\{0^{b}\right\}$;

- For $i=1, \ldots, \lambda$ : for any $(s, t) \in \mathcal{Q}$ and any $L$ such that $s \oplus\left(L \| 0^{c_{\mathrm{ab}}}\right) \in V_{i-1}$, vertex $t$ is added to $V_{i}$ and arrow $s \oplus\left(L \| 0^{c_{\mathrm{ab}}}\right) \stackrel{L}{\longrightarrow} t$ with label $L$ is added to $A$.

For given $c_{\mathrm{ab}}, \lambda$, and $\mathcal{Q}$, the size of the yield $\left|\operatorname{yield}_{c_{\mathrm{ab}}, \lambda}(\mathcal{Q})\right|$ equals the number of paths from $V_{0}=\left\{0^{b}\right\}$ to $V_{\lambda}$ in $G_{c_{\mathrm{ab}}, \lambda}(\mathcal{Q})$.

A visualization of the graph is depicted in Figure 2. By construction, $\left|V_{i}\right| \leq|\mathcal{Q}|$ for all $i=1, \ldots, \lambda$. We highlight three further properties of the graph $G_{c_{\mathrm{ab}}, \lambda}(\mathcal{Q})$ :

1. Two arrows departing from the same node, e.g., from $u$ in Figure 2, happens if there exist distinct $L, L^{\prime} \in\{0,1\}^{r_{\mathrm{ab}}}$ and distinct $t, t^{\prime} \in\{0,1\}^{b}$ such that

$$
\left(u \oplus\left(L \| 0^{c_{\mathrm{ab}}}\right), t\right),\left(u \oplus\left(L^{\prime} \| 0^{c_{\mathrm{ab}}}\right), t^{\prime}\right) \in \mathcal{Q} .
$$

If the adversary makes a forward query, it can set such case with probability 1 ; if it makes an inverse query, the case happens only if the query response $s$ matches that of an existing vertex $u$ in the previous layer: $\lfloor s\rfloor_{c_{\mathrm{ab}}}=\lfloor u\rfloor_{c_{\mathrm{ab}}}$;

\footnotetext{
${ }^{2}$ Technically, $V$ is a set of vertex sets.
} 


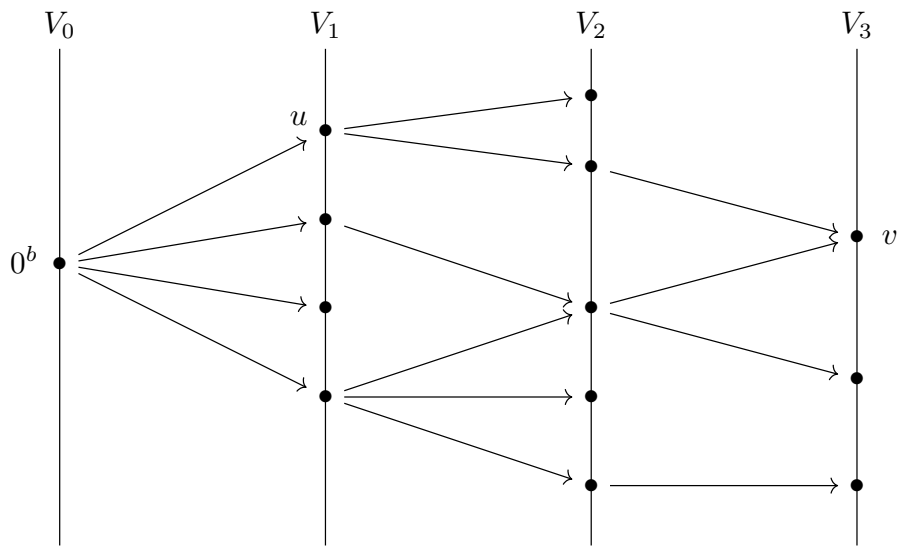

Figure 2: Example graph $G_{c_{\mathrm{ab}}, 3}(\mathcal{Q})$ consisting of 4 vertex layers. Edge and vertex labels are omitted for clarity, except for vertices $u$ and $v$ for the sake of discussion.

2. Two arrows arriving at the same node, e.g., at $v$ in Figure 2, happens if there exist distinct $L, L^{\prime} \in\{0,1\}^{r_{\mathrm{ab}}}$ and distinct $s, s^{\prime} \in\{0,1\}^{b}$ such that

$$
\left(s \oplus\left(L \| 0^{c_{\mathrm{ab}}}\right), v\right),\left(s^{\prime} \oplus\left(L^{\prime} \| 0^{c_{\mathrm{ab}}}\right), v\right) \in \mathcal{Q} .
$$

(These two queries are necessarily the same as $\pi$ is a permutation.) If the adversary makes an inverse query, it can set such case with probability 1 (but the new edge will likely not appear in the tree, as the tree is built up from $0^{b}$ in $V_{0}$ ); if it makes a forward query, the case happens only if there exist two nodes in the previous layer with equal capacity: $\lfloor s\rfloor_{c_{\mathrm{ab}}}=\left\lfloor s^{\prime}\right\rfloor_{c_{\mathrm{ab}}}$;

3. As $\mathcal{Q}$ is a transcript of permutation queries, we can observe the following. In the first of the above properties, the two vertices at which the arrows end, $t$ and $t^{\prime}$, must necessarily be distinct; in the second case, the two vertices from which the arrows depart, $s$ and $s^{\prime}$, must necessarily be distinct. We can particularly conclude that there do not exist layer $i \in\{1, \ldots, \lambda\}$ and vertices $v_{i-1} \in V_{i-1}$ and $v_{i} \in V_{i}$ such that $G_{c_{\mathrm{ab}, \lambda}}(\mathcal{Q})$ contains two arrows from $v_{i-1}$ to $v_{i}$.

We regularly talk about configurations of paths in said graph.

Definition 2. Consider GKS based on $\pi \in \operatorname{perm}(b)$, and let $\mathcal{Q}$ be a transcript of $\pi$. Consider graph $G_{c_{\mathrm{ab}}, \lambda}(\mathcal{Q})$. The configuration of a path from $v_{0} \in V_{0}$ to $v_{\lambda} \in V_{\lambda}$ is defined as a tuple $C=\left(C_{1}, \ldots, C_{\lambda}\right) \in\{0,1\}^{\lambda}$, where $C_{i}=0$ if the arrow from $V_{i-1}$ to $V_{i}$ corresponds to a forward query and $C_{i}=1$ if it corresponds to an inverse query.

\section{Key Prediction}

For $F \in\{\mathrm{GKS}, \mathrm{OKS}, \mathrm{FKS}\}$, we define key prediction security by the following experiment: for a random permutation $\pi \stackrel{\$}{\leftarrow}$ perm $(b)$, consider an adversary $\mathcal{A}$ that has oracle access to $\pi^{ \pm}$, meaning that it can query $\pi$ in forward and inverse direction. The adversary can make a finite amount of queries to its oracle, which are summarized in a transcript $\mathcal{Q}$. Then, a key $K \stackrel{\$}{\leftarrow}\{0,1\}^{k}$ is uniformly randomly drawn, and the adversary wins if $K \in \operatorname{yield}_{c_{\mathrm{ab}, \lambda}}(\mathcal{Q})$. Formally: 
Definition 3. The key prediction security of $F \in\{\mathrm{GKS}, \mathrm{OKS}, \mathrm{FKS}\}$ against an adversary $\mathcal{A}$ is defined as

$$
\operatorname{Adv}_{F}^{\text {key-pre }}(\mathcal{A})=\operatorname{Pr}\left(\pi \stackrel{\$}{\leftarrow} \operatorname{perm}(b), \mathcal{Q} \leftarrow \mathcal{A}^{\pi^{ \pm}}, K \stackrel{\$}{\leftarrow}\{0,1\}^{k}: K \in \operatorname{yield}_{c_{\mathrm{ab}, \lambda}}(\mathcal{Q})\right) .
$$

For $N \geq 0$, we define by $\operatorname{Adv}_{F}^{\text {key-pre }}(N)$ the maximum over all adversaries making $N$ queries to its oracle.

In above game, the key is only drawn after the adversary queries its oracle $\pi^{ \pm}$. Its attack tools are mere combinatorics: it maximizes its chances by maximizing the size of the yield of the query transcript.

In the remainder of the section, we will focus on $F=\mathrm{OKS}$, hence we write $\left(c_{\mathrm{ab}}, r_{\mathrm{ab}}\right)=$ $\left(c_{\mathrm{sq}}, r_{\mathrm{sq}}\right)=:(c, r)$. We will recall the bound of Gaži et al. [GPT15a, GPT15b] on Adv $\mathbf{A v y}_{\mathrm{OKS}}$ in Section 3.1, and present our improved result in Section 3.2. Sections 3.3 and 3.4 cover the rationale and proof of our result, respectively. We discuss the possibility to generalize our results to multi-key security in Section 3.5.

\subsection{Bound of Gaži et al.}

We briefly discuss the bound of Gaži et al. [GPT15b, Lemma 12] (full version of [GPT15a])

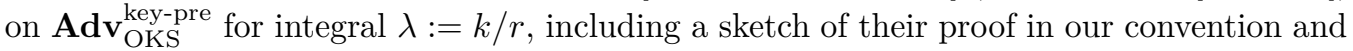
notation. Afterwards, we will highlight several aspects of their analysis that contribute to non-tightness of their bound.

Proposition 1 (Gaži et al. [GPT15b, Lemma 12]). Consider F = OKS for parameters $(b, c, r, k)$, where $\lambda:=k / r$ is integral. We have

$$
\operatorname{Adv}_{F}^{\text {key-pre }}(N) \leq\left\{\begin{array}{l}
\frac{N}{2^{k}}, \text { if } \lambda=1, \\
\min \left\{\frac{N^{2}}{2^{c}}+\frac{N}{2^{k}}, \frac{1}{2^{b}}+\frac{N 2^{\lambda}(3 b-1)^{\lambda / 2}}{2^{k / 2}}\right\}, \text { if } \lambda>1 .
\end{array}\right.
$$

Proof (sketch). In case of $\lambda=1$, the adversary can make at most $N$ evaluations of $\pi$ for different key guesses $K_{1}, \ldots, K_{N}$, hence can obtain yield of size at most $\left|\operatorname{yield}_{c, \lambda}(\mathcal{Q})\right| \leq N$. Alternatively, the claim follows from the fact that $\left|V_{1}\right| \leq|\mathcal{Q}|$ and that $G_{c, \lambda}(\mathcal{Q})$ contains no two arrows from $0^{b} \in V_{0}$ to one and the same node in $V_{1}$ (property 3 in Section 2.2). The probability that a randomly selected key $K \stackrel{\$}{\leftarrow}\{0,1\}^{k}$ is in the yield is thus at most $N / 2^{k}$.

We sketch Gaži et al.'s approach for $\lambda \geq 2$, assuming for simplicity that $k=\lambda \cdot r$. Consider any adversary making $N$ queries, stored in a transcript $\mathcal{Q}$. For $\alpha \in \mathbb{N}$, define by $\mathrm{mc}_{\alpha}$ the event that there exist no $\alpha+1$ queries $\left(s_{i}, t_{i}\right)_{i=1}^{\alpha+1}$ to $\pi$ for which either all queries are in forward direction and $\left\lfloor t_{1}\right\rfloor_{c}=\cdots=\left\lfloor t_{\alpha+1}\right\rfloor_{c}$, or all are in inverse direction and $\left\lfloor s_{1}\right\rfloor_{c}=\cdots=\left\lfloor s_{\alpha+1}\right\rfloor_{c}$. Obviously, $\operatorname{Pr}\left(\neg \mathrm{mc}_{1}\right) \leq N^{2} / 2^{c}$. In addition, Gaži et al. prove using the Chernoff bound that: ${ }^{3}$

$$
\operatorname{Pr}\left(\neg \mathrm{mc}_{3 b-1}\right) \leq 1 / 2^{b}
$$

Gaži et al. subsequently prove that

$$
\begin{aligned}
\mathrm{mc}_{1} & \Longrightarrow\left|\operatorname{yield}_{c, \lambda}(\mathcal{Q})\right| \leq N \\
\mathrm{mc}_{3 b-1} & \Longrightarrow\left|\operatorname{yield}_{c, \lambda}(\mathcal{Q})\right| \leq N 2^{\lambda}(3 b-1)^{\lambda / 2} 2^{k / 2},
\end{aligned}
$$

which in turn concludes the proof as the probability that a randomly selected key $K \stackrel{\$}{\leftarrow}$ $\{0,1\}^{k}$ is in the yield is at most $\left|\operatorname{yield}_{c, \lambda}(\mathcal{Q})\right| / 2^{k}$. It thus remains to prove (9) and (10).

\footnotetext{
${ }^{3}$ There's a small gap in the reasoning, namely that the Chernoff bound considers a sum of independent events whereas in the current case a sum of inherently dependent events is considered.
} 
Regarding (9), mc 1 implies that $G_{c, \lambda}(\mathcal{Q})$ is a tree (cf. [GPT15b, Lemma 12]), and $\left|\operatorname{yield}_{c, \lambda}(Q)\right|$ is at most the number of nodes at layer $\lambda$ : $N$. Regarding (10), as the adversary only makes $N$ queries, there are at most $N$ possible nodes in $V_{\lambda}$ at distance $\lambda$ from $0^{b}$. For any such node $v_{\lambda}$, there are $\lambda$ primitive queries connecting

$$
0^{b} \longleftrightarrow \cdots \longleftrightarrow v_{\lambda}
$$

and each of these primitive queries could have been made in forward or in inverse direction. Let $C \in\{0,1\}^{\lambda}$ be any configuration, where $C_{i}=0$ means that the arrow from $V_{i-1}$ to $V_{i}$ corresponds to a forward query and $C_{i}=1$ that it corresponds to an inverse query (see Definition 2). There are $2^{\lambda}$ possible configurations. Starting from the end node, by $\mathrm{mc}_{3 b-1}$ there are at most $3 b-1$ arrows into $v_{\lambda}$ that correspond to forward queries to $\pi$, but we do not know anything about inverse queries, other than that the in-degree of $v_{\lambda}$ is at most $2^{r}$. This gives the following upper bound on the number of paths from $0^{b}$ to (fixed) $v_{\lambda}$ for fixed configuration $C$ :

$$
(3 b-1)^{\lambda-|C|}\left(2^{r}\right)^{|C|} .
$$

For $|C| \leq \lambda / 2$ this term is at most $(3 b-1)^{\lambda / 2}\left(2^{r}\right)^{\lambda / 2}$ as $3 b-1 \leq 2^{r}$. For $|C|>\lambda / 2$ one can reverse the reasoning, i.e., start from the first node $0^{b}$ instead of end node $v_{\lambda}$. This likewise gives upper bound

$$
(3 b-1)^{|C|}\left(2^{r}\right)^{\lambda-|C|},
$$

which for $|C|>\lambda / 2$ is also at $\operatorname{most}(3 b-1)^{\lambda / 2}\left(2^{r}\right)^{\lambda / 2}$ as $3 b-1 \leq 2^{r}$. Summing over all possible configurations $C$ and all possible end nodes, we obtain

$$
\mid \text { yield }_{c, \lambda}(\mathcal{Q}) \mid \leq N 2^{\lambda}(3 b-1)^{\lambda / 2}\left(2^{r}\right)^{\lambda / 2}=N 2^{\lambda}(3 b-1)^{\lambda / 2} 2^{k / 2},
$$

as $k=\lambda \cdot r$.

Besides the minor and insignificant glitch that the Chernoff bound is applied to the sum of dependent events, the proof has two shortcomings that yield non-tightness of the bound:

(i) The proof approach selects an end node and then upper bounds the amount of vertices at the different layers. The selection of the end node ( $N$ possibilities), however, already fixes the last vertex. A single query may connect multiple elements from $V_{\lambda-1}$ with $V_{\lambda}$, hence one cannot just assume that fixing the end node gives one arrow "for free," but it still fixes the inner part of the node at layer $V_{\lambda-1}$;

(ii) Assuming $\mathrm{mc}_{3 b-1}$, there is no $3 b$-fold inner collision, and no more than $3 b-1$ forward queries that map to the same node in a layer $V_{i}$; yet for the inverse queries Gaži et al. cannot rely on $\mathrm{mc}_{3 b-1}$ and resort to the fact that any node has at most $2^{r}$ incoming arrows. It is intuitively appealing to say that also for inverse queries the number of useful arrows is at most $3 b-1$, where useful refers to the fact that the path should lead to root $0^{b} \in V_{0}$.

\subsection{Improved Key Prediction Security}

We derive the following improved bound for key prediction security. The bound for $\lambda=1$ of Proposition 1 is already tight, and we restrict ourselves to the case of $\lambda>1$.

Theorem 1. Consider $F=$ OKS for parameters $(b, c, r, k)$, where $\lambda:=\lceil k / r\rceil>1$. Let $\alpha>\lambda$ be a natural number. Let $0 \leq N \leq 2^{b-1}$. We have

$$
\operatorname{Adv}_{F}^{\text {key-pre }}(N) \leq \frac{(2 \alpha)^{\lambda-1} N}{2^{k}}+\lambda 2^{\lambda} 2^{2 c}\left(\frac{2 e N^{*}}{2^{c} \alpha}\right)^{\alpha / \lambda}
$$

where $N^{*}=\max \left\{N, \lambda 2^{(\lambda-1) r}\right\}$. 


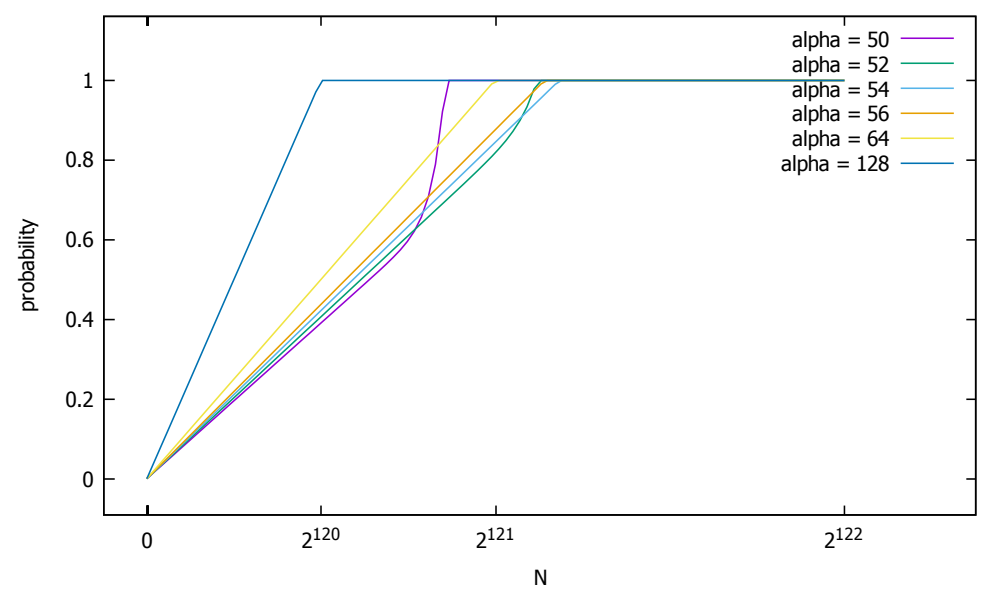

Figure 3: For parameter set $(b, c, r, k)=(192,128,64,128)$, the bound of Theorem 1 for $\alpha=50,52,54,56,64,128$ (from right to left).

The proof will be given in Section 3.4, preceded by its rationale in Section 3.3.

Parameter $\alpha$ is a threshold: the first term increases whereas the second term decreases for increasing $\alpha$. By taking $\alpha / \lambda \geq c$, we obtain that $2^{\lambda} 2^{2 c} \leq 2^{k} 2^{2 c} \leq 2^{3 c} \leq 8^{\alpha / \lambda}$, and above bound simplifies to

$$
\operatorname{Adv}_{F}^{\text {key-pre }}(N) \leq \frac{(2 \alpha)^{\lambda-1} N}{2^{k}}+\lambda\left(\frac{16 e N^{*}}{2^{c} \alpha}\right)^{\alpha / \lambda}
$$

For $N$ close to $2^{k}, N^{*}=N$ and the first term dominates the equation. For smaller $N$, $N^{*}=\lambda 2^{(\lambda-1) r}$, and the second term dominates.

For the case of $(b, c, r, k)=(192,128,64,128)$, with $\lambda=2$, the graph in Figure 3 plots the bound of Theorem 1 for various choices of $\alpha$. One sees that taking $\alpha \geq 2 c$ is convenient for making the bound simple, but it is a rough estimate. Better choices of $\alpha$, less than $2 c$, yield a stronger bound. The first term of (11) dominates as long as $\alpha \geq 54$. For $\alpha \leq 53$ the second term in (11) starts to dominate. The graph in Figure 3 depicts it well for $\alpha=52$ : it follows the linear behavior of the first term, until at some point the exponential term of (11) becomes dominating. Numerical computation shows that for $\alpha=54$, the bound of (11) equals 1 for $N \approx 2^{121.25}$.

For the example outlined above, the bound of Gaži et al. of Proposition 1 equals 1 for $N^{2}+N \approx 2^{128}$. The best known attack is the generic one, that fixes $\lambda-1$ blocks $K_{1}, \ldots, K_{\lambda-1}$ and varies $K_{\lambda}$ : this procedure renders a yield of size exactly $N-(\lambda-1)$ and succeeds in predicting the key with probability $(N-(\lambda-1)) / 2^{k}$. A comparison of the old bound, the new bound (for $\alpha=54$ ), and the best known attack is given in Figure 4 . The bound of Theorem 1 permits a small loss in comparison with the best known attack, which comes from the accidental event of multi-collisions on the inner part of $\pi$. The term increases exponentially in $\lambda$, which is due to the fact that multi-collisions could occur at every evaluation of $\pi$, and they can amplify each other. In the end, however, it only yields a small loss.

\subsection{Rationale Behind Theorem 1}

The proof, at a high level, relies on the observation that any path from $V_{0}$ to a fixed vertex $v_{\lambda} \in V_{\lambda}$ must contain at least one collision on the inner part: either between a query and $0^{b} \in V_{0}$ or $v_{\lambda}$, or between two queries at any layer in $V_{1}, \ldots, V_{\lambda-1}$. In addition, it uses that for a fixed inner part, there are at $\operatorname{most} \min \left\{N, 2^{r}\right\}$ queries. 


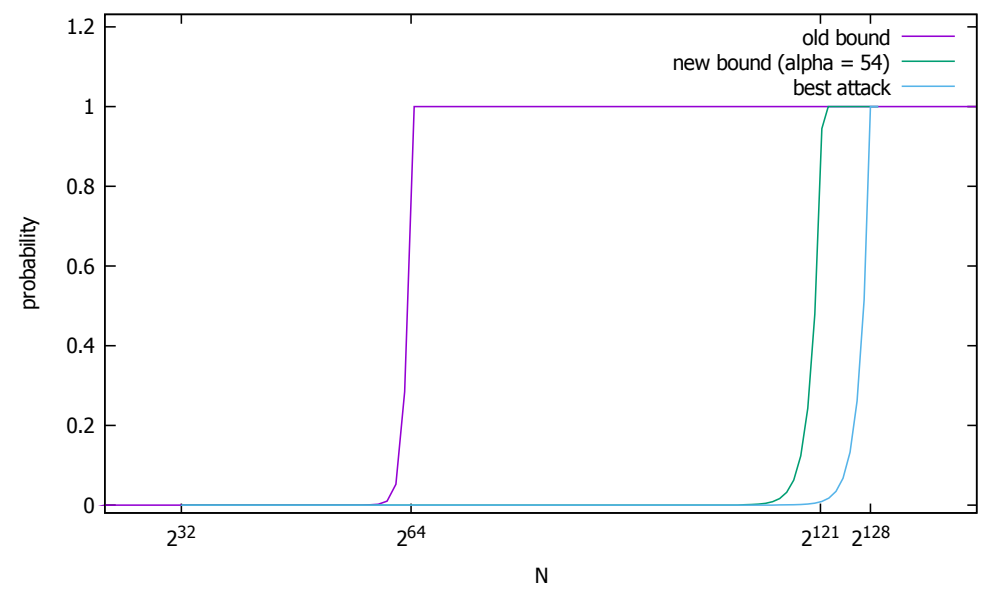

Figure 4: For parameter set $(b, c, r, k)=(192,128,64,128)$, the old bound of Proposition 1 , the new bound of Theorem 1 for $\alpha=54$, and the best known attack (from left to right). The $N$ axis is in logarithmic scale.

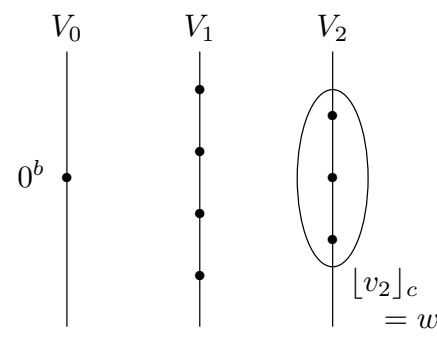

(a) Targeted subgraph consisting of 2 layers, where $w \in\{0,1\}^{c}$ is fixed.

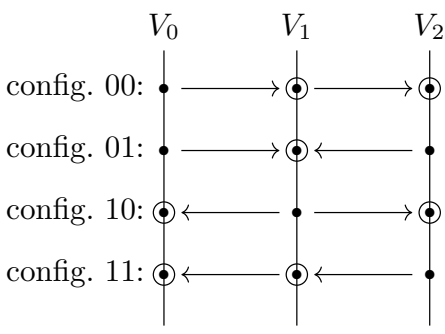

(b) Possible configurations for the directions of the queries. A circle $\bigcirc$ indicates that a fixed inner value must be hit.

Figure 5: Graph and configurations for the rationale of Section 3.3.

The proof is easiest understood by considering $\lambda=3$. In this case, we consider graph $G_{c, 3}(\mathcal{Q})$ consisting of four layers $V_{0}, V_{1}, V_{2}, V_{3}$. The first step in the proof will be to fix the last vertex $v_{3} \in V_{3}$. There are at most $N$ choices, as this choice is equivalent to fixing $\left(s, v_{3}\right) \in \mathcal{Q}$. The choice also fixes the inner part of the nodes in $V_{2}$ under consideration: the query links $V_{2}$ with $V_{3}$ only for nodes $v_{2}$ such that $\left\lfloor v_{2}\right\rfloor_{c}=\lfloor s\rfloor_{c}$. Define the fixed capacity by $w:=\lfloor s\rfloor_{c} \in\{0,1\}^{c}$. We henceforth have to focus on paths from $0^{b} \in V_{0}$ to any $v_{2} \in V_{2}$, where $\left\lfloor v_{2}\right\rfloor_{c}=w$. See also Figure 5a. Any of these paths fits one of the four configurations of Figure 5b.

Assume, for the sake of argument, that $\mathcal{Q}$ contains no $(\alpha+1)$-fold inner collision (in either forward or inverse direction). For configuration 00 of Figure 5 b, we have at most $\alpha$ arrows from $V_{1}$ to $V_{2}$, each of which fixes the inner part of the node in $V_{1}$, yielding at most $\alpha$ arrows from $V_{0}$ to $V_{1}$; see also the circle indications in the figure. We have obtained that there are at most $\alpha^{2}$ paths that obey to configuration 00. Likewise, there are at most $\alpha^{2}$ paths for configurations 10 and 11 , using that the node in $V_{0}, 0^{b}$, has its inner part fixed: $0^{c}$. What remains is configuration 01: the inner parts of the values at layers $V_{0}$ and $V_{2}$ are fixed, and thus there are at most $\min \left\{N, 2^{r}\right\}$ queries in $\mathcal{Q}$ that could depart from $V_{0}$ in forward direction and $V_{2}$ in inverse direction. The expected numbers of collisions in the 
middle is

$$
\frac{\min \left\{N, 2^{r}\right\}^{2}}{2^{c}}
$$

By the Markov inequality, more than $\alpha^{2}$ paths exist with probability at most

$$
\frac{\min \left\{N, 2^{r}\right\}^{2}}{2^{c} \alpha^{2}}
$$

This gives $\left|\operatorname{yield}_{c, 3}(\mathcal{Q})\right|=4 \alpha^{2} N$, except with probability (12) and the probability that there exists an $(\alpha+1)$-fold inner collision. Above reasoning also pinpoints the technical difference between our proof and that of Gaži et al. (Proposition 1): we more accurately bound the number of paths in order to obtain a tighter yield.

Unfortunately, above reasoning is not entirely correct: the evaluation of configuration 01 has to be performed for all possible $w \in\{0,1\}^{c}$, as the adversary can freely choose the query for the last layer. Multiplying (12) by $2^{c}$ does not help; to the contrary, the bound becomes meaningless. Instead, configuration 01 will also be analyzed by relying on the non-existence of $(\alpha+1)$-fold inner collisions: any hit adds at most $\alpha$ solutions.

Additional issues surface if we extend the reasoning to larger values of $\lambda$ : configurations like $001\left(0^{c} \rightarrow \cdot \rightarrow \cdot \leftarrow w\right)$ or $0101\left(0^{c} \rightarrow \cdot \leftarrow \cdot \rightarrow \cdot \leftarrow w\right)$ appear. To resolve these issues, a recursive reasoning (in $\lambda$ ) will be performed.

\subsection{Proof of Theorem 1}

Consider $F=$ OKS for parameters $(b, c, r, k)$, where $\lambda:=\lceil k / r\rceil>1$, and let $\pi \stackrel{\$}{\leftarrow} \operatorname{perm}(b)$. Consider any adversary $\mathcal{A}$ with two-sided query access to $\pi$. We will record the query history in a transcript $\mathcal{Q}$, where we keep track of the query direction using a bit $C \in\{0,1\}$, i.e., a tuple $(C, s, t) \in \mathcal{Q}$ is made in forward direction if $C=0$ and inverse direction if $C=1$. Associated with $\mathcal{Q}$ is graph $G_{c, \lambda}(\mathcal{Q})=(V, A)$ as defined in Section 2.2.

\subsubsection{Defining Auxiliary Events}

Let $\alpha>0$ be an integral threshold. At the core of our proof is the event $\mathrm{mc}$, that bounds the maximum size of a multi-collision in $\mathcal{Q}$. We define

$$
\mathrm{mc}=\mathrm{mc}^{+} \wedge \mathrm{mc}^{-},
$$

where

$$
\begin{gathered}
\mathrm{mc}^{+}: \max _{w \in\{0,1\}^{c}}\left|\left\{(0, s, t) \in \mathcal{Q} \mid\lfloor t\rfloor_{c}=w\right\}\right| \leq \alpha, \\
\mathrm{mc}^{-}: \max _{v \in\{0,1\}^{c}}\left|\left\{(1, s, t) \in \mathcal{Q} \mid\lfloor s\rfloor_{c}=v\right\}\right| \leq \alpha .
\end{gathered}
$$

The event $\mathrm{mc}$ corresponds to $\mathrm{mc}_{\alpha}$ in the proof of Proposition 1, be it with $\alpha$ omitted as subscript.

The proof will, implicitly, be performed by induction on $\lambda$. For $i=1, \ldots, \lambda-1$, we define the event $\mathrm{ch}_{i}$, a condition on chains of length $i$, as follows:

$$
\begin{aligned}
\operatorname{ch}_{i}: \max _{C \in\{0,1\}^{i}} \max _{v, w \in\{0,1\}^{c}} \mid\left\{\left(C_{1}, s_{1}, t_{1}\right), \ldots,\left(C_{i}, s_{i}, t_{i}\right) \in \mathcal{Q} \mid\right. \\
\left.\left\lfloor s_{1}\right\rfloor_{c}=v \wedge\left(\left\lfloor t_{j}\right\rfloor_{c}=\left\lfloor s_{j+1}\right\rfloor_{c}\right)_{j=1}^{i-1} \wedge\left\lfloor t_{i}\right\rfloor_{c}=w\right\} \mid \leq \alpha^{i} .
\end{aligned}
$$

In other words, $\mathrm{ch}_{i}$ is the maximum number of solutions to

$$
v \stackrel{C_{1}}{\longleftrightarrow} \cdot \stackrel{C_{2}}{\longleftrightarrow} \cdots \stackrel{C_{i}}{\longleftrightarrow} w
$$


maximized over all possible configurations $C=\left(C_{1}, \ldots, C_{i}\right) \in\{0,1\}^{i}$ (the label on the arrow indicates the configuration of the query, see also Definition 2) and start and end nodes $v, w \in\{0,1\}^{c}$. There is an important difference between $\mathrm{ch}_{1}$ ( or $\mathrm{ch}_{i}$ in general) on the one hand and $\mathrm{mc}$ on the other hand: in $\mathrm{ch}_{1}$ the inner values of both sides of the path are fixed, whereas in $\mathrm{mc}$ only one side is fixed. Nevertheless, $\mathrm{mc} \Rightarrow \mathrm{ch}_{1}$ by definition. Furthermore, for $i=\lambda-1$ the proof needs $\mathrm{ch}_{\lambda-1}$ only for $v=0^{c}$, but we have opted to include the general event for simplicity of argument.

\subsubsection{Main Step}

The main step in bounding $\mathbf{A d v}_{F}^{\text {key-pre }}(N)$ is the following:

$$
\operatorname{Adv}_{F}^{\text {key-pre }}(N) \leq \operatorname{Pr}\left(K \stackrel{\$}{\leftarrow}\{0,1\}^{k}: K \in \operatorname{yield}_{c_{\mathrm{ab}}, \lambda}(\mathcal{Q}) \mid \operatorname{ch}_{\lambda-1}\right)+\operatorname{Pr}\left(\neg \mathrm{ch}_{\lambda-1}\right) .
$$

Note that this probability reduction only uses event $\mathrm{ch}_{\lambda-1}$ explicitly: under the condition that this event holds, a proper bound on $\left|\operatorname{yield}_{c, \lambda}(\mathcal{Q})\right|$ will be derived. This derivation, i.e., a bound on the first probability of (18), will be given in Section 3.4.3. The analysis that $\mathrm{ch}_{\lambda-1}$ is violated, the second probability of (18), is inductive, and relies on the condition that none of the "shorter" events $\mathrm{ch}_{\lambda-2} \wedge \cdots \wedge \mathrm{ch}_{1} \wedge \mathrm{mc}$ is violated. A bound on this probability will be derived in Section 3.4.4.

\subsubsection{Bounding the Yield}

Fix any vertex $v_{\lambda} \in V_{\lambda}$. There are at most $N$ choices as this choice is equivalent to fixing $\left(s, v_{\lambda}\right) \in \mathcal{Q}$, and it also fixes the inner part $w:=\lfloor s\rfloor_{c} \in\{0,1\}^{c}$ for the node at shore $V_{\lambda-1}$. We thus have to focus on paths from $0^{b} \in V_{0}$ to any $v_{\lambda-1} \in V_{\lambda-1}$ with $\left\lfloor v_{\lambda-1}\right\rfloor_{c}=w$. Let $C \in\{0,1\}^{\lambda-1}$ be any configuration. By $\mathrm{ch}_{\lambda-1}$, there are at most $\alpha^{\lambda-1}$ paths from $0^{c}$ to the fixed $w$ for this particular configuration. Summing over all possible configurations and all possible choices $v_{\lambda}$, we obtain that

$$
\operatorname{ch}_{\lambda-1} \Longrightarrow\left|\operatorname{yield}_{c, \lambda}(\mathcal{Q})\right| \leq(2 \alpha)^{\lambda-1} N \text {. }
$$

We obtain for the first probability of (18):

$$
\operatorname{Pr}\left(K \stackrel{\$}{\leftarrow}\{0,1\}^{k}: K \in \operatorname{yield}_{c_{\mathrm{ab}}, \lambda}(\mathcal{Q}) \mid \operatorname{ch}_{\lambda-1}\right)=\frac{\left|\operatorname{yield}_{c, \lambda}(\mathcal{Q})\right|}{2^{k}} \leq \frac{(2 \alpha)^{\lambda-1} N}{2^{k}}
$$

\subsubsection{Analyzing Auxiliary Events}

It remains to analyze the second probability of (18). By basic probability theory,

$$
\operatorname{Pr}\left(\neg \mathrm{ch}_{\lambda-1}\right) \leq \operatorname{Pr}(\neg \mathrm{mc})+\sum_{i=1}^{\lambda-1} \operatorname{Pr}\left(\neg \mathrm{ch}_{i} \mid \mathrm{ch}_{i-1} \wedge \cdots \wedge \mathrm{ch}_{1} \wedge \mathrm{mc}\right)
$$

We will analyze the probabilities in Lemmas 1 and 2 below. After the proof of Lemma 2, we will combine the results into a clean and concise bound on (20).

Lemma 1. Consider $F=$ OKS for parameters $(b, c, r, k)$. Let $\alpha>\lambda:=\lceil k / r\rceil>1$ be a natural number. Consider any adversary playing the key prediction security game of Definition 3 against $F=\mathrm{OKS}$, that makes $N \leq 2^{b-1}$ queries recorded in a transcript $\mathcal{Q}$. Then,

$$
\operatorname{Pr}(\neg \mathrm{mc}) \leq 2 \cdot 2^{c}\left(\frac{2 e N}{2^{c} \alpha}\right)^{\alpha}
$$

where the event mc is defined in Section 3.4.1. 
Proof (of Lemma 1). Without loss of generality, consider $\mathrm{mc}^{+}$. Fix any $w \in\{0,1\}^{c}$. Any forward query $(0, s, t)$ satisfies $\lfloor t\rfloor_{c}=w$ with probability at most $2^{r} /\left(2^{b}-N\right)$, as the response is randomly drawn from a set of size at least $2^{b}-N$ and at most $2^{r}$ of those fulfill the condition. More than $\alpha$ satisfy the condition with probability at most ${ }^{4}$

$$
\left(\begin{array}{l}
N \\
\alpha
\end{array}\right)\left(\frac{2^{r}}{2^{b}-N}\right)^{\alpha} \leq\left(\frac{2 e N}{2^{c} \alpha}\right)^{\alpha}
$$

using a well-known upper bound on binomial coefficients, and the assumption that $N \leq 2^{b-1}$. The proof is completed by summing over all possible $w \in\{0,1\}^{c}$ and by taking into account $\mathrm{mc}^{-}$as well (cf. (13)).

Lemma 2. Consider $F=$ OKS for parameters $(b, c, r, k)$. Let $\alpha>\lambda:=\lceil k / r\rceil>1$ be $a$ natural number. Let $i \in\{1, \ldots, \lambda-1\}$. Consider any adversary playing the key prediction security game of Definition 3 against $F=\mathrm{OKS}$, that makes $N \leq 2^{b-1}$ queries recorded in a transcript $\mathcal{Q}$. Then,

$$
\operatorname{Pr}\left(\neg \mathrm{ch}_{i} \mid \mathrm{ch}_{i-1} \wedge \cdots \wedge \mathrm{ch}_{1} \wedge \mathrm{mc}\right) \leq\left\{\begin{array}{l}
0, \text { for } i=1, \\
i 2^{i} 2^{2 c}\left(\frac{2 e i 2^{i r}}{2^{c} \alpha}\right)^{\alpha / i}, \text { for } i=2, \ldots, \lambda-1,
\end{array}\right.
$$

where the events $\mathrm{ch}_{i}(i=1, \ldots, \lambda-1)$ and $\mathrm{mc}$ are defined in Section 3.4.1.

Proof (of Lemma 2). We have $\mathrm{mc} \Rightarrow \mathrm{ch}_{1}$ by definition, and focus on the case of arbitrary $i>1$. Fix any configuration $C \in\{0,1\}^{i}$ and any $v, w \in\{0,1\}^{c}$, in total $2^{i} 2^{2 c}$ possible choices. We aim to prove that the number of solutions to (17) is at most $\alpha^{i}$. To the contrary, assume it is more than $\alpha^{i}$. Then, by the pigeonhole principle, there must be an index $j \in\{1, \ldots, i\}$ such that more than $\alpha^{i} / i$ solutions are constituted with the winning query, i.e., the query that completes the chain, occurring at position $j .{ }^{5}$ Without loss of generality (the argument is fully symmetric), assume that $C_{j}=0$, i.e., that it is a forward query.

As $v \in\{0,1\}^{c}$ is fixed, there are at most $2^{j r}$ possible values $s \in\{0,1\}^{b}$ at distance $j-1$ from $v$, i.e., the adversary can make at most $\min \left\{N, 2^{j r}\right\}$ attempts. As $w \in\{0,1\}^{c}$ is fixed, there are at most $2^{(i-j) r}$ possible inner parts $x$ such that a path

$$
x \stackrel{C_{j+1}}{\longleftrightarrow} \cdots \stackrel{C_{i}}{\longleftrightarrow} w
$$

can be constituted from the transcript. The new forward query hits any of these inner parts with probability at most $2^{(i-j) r} \cdot 2^{r} /\left(2^{b}-N\right)$. By $\mathrm{ch}_{j-1}$ and $\mathrm{ch}_{i-j}$, any such hit adds at most $\alpha^{j-1} \cdot \alpha^{i-j}=\alpha^{i-1}$ solutions. In order to get more than $\alpha^{i} / i$ solutions, there must be more than $\alpha / i$ collisions, which happens with probability at most

$$
\left(\begin{array}{c}
\min \left\{N, 2^{j r}\right\} \\
\alpha / i
\end{array}\right)\left(\frac{2^{(i-j) r} \cdot 2^{r}}{2^{b}-N}\right)^{\alpha / i} \leq\left(\frac{2 e i 2^{i r}}{2^{c} \alpha}\right)^{\alpha / i}
$$

again by bounding the binomial coefficient and using the assumption that $N \leq 2^{b-1}$, and where we recall that the adversary has only $\min \left\{N, 2^{j r}\right\} \leq 2^{j r}$ shots to success. The proof is completed by summing over all possible configurations $C \in\{0,1\}^{i}$, start and end nodes $v, w \in\{0,1\}^{c}$, and positions of the winning query $j \in\{1, \ldots, i\}$.

\footnotetext{
${ }^{4}$ In the bound we could take $\alpha+1$ instead of $\alpha$, but have opted not to do so for simplicity.

${ }^{5}$ It could be that this query occurs at multiple positions in the chain, but this does not invalidate the reasoning due to generous counting.
} 
From (20) and Lemmas 1 and 2 we immediately obtain, using that $\alpha>\lambda$,

$$
\begin{aligned}
\operatorname{Pr}\left(\neg \mathrm{ch}_{\lambda-1}\right) & \leq 2 \cdot 2^{c}\left(\frac{2 e N}{2^{c} \alpha}\right)^{\alpha}+\sum_{i=2}^{\lambda-1} i 2^{i} 2^{2 c}\left(\frac{2 e i 2^{i r}}{2^{c} \alpha}\right)^{\alpha / i} \\
& \leq 2 \cdot 2^{c}\left(\frac{2 e N}{2^{c} \alpha}\right)^{\alpha}+\sum_{i=2}^{\lambda-1} \lambda 2^{i} 2^{2 c}\left(\frac{2 e \lambda 2^{(\lambda-1) r}}{2^{c} \alpha}\right)^{\alpha / \lambda} \\
& \leq \sum_{i=1}^{\lambda-1} \lambda 2^{i} 2^{2 c}\left(\frac{2 e N^{*}}{2^{c} \alpha}\right)^{\alpha / \lambda} \\
& \leq \lambda 2^{\lambda} 2^{2 c}\left(\frac{2 e N^{*}}{2^{c} \alpha}\right)^{\alpha / \lambda}
\end{aligned}
$$

where $N^{*}=\max \left\{N, \lambda 2^{(\lambda-1) r}\right\}$. Slight improvements in the bound could be achieved, at the cost of readability penalties, in the derivation of Lemma 2 and in the bounding of (24) above.

\subsection{Generalization to Multi-Key Security}

It is straightforward to generalize our analysis to multi-key security. First, to generalize Definition 3 , one would consider $K_{1}, \ldots, K_{u} \stackrel{\$}{\leftarrow}\{0,1\}^{k}$, where $u \in \mathbb{N}$ is the number of users, and the adversary wins if $K_{i} \in \operatorname{yield}_{c_{\mathrm{ab}}, \lambda}(\mathcal{Q})$ for some $i \in\{1, \ldots, u\}$ :

Definition 4. Let $u \in \mathbb{N}$. The $u$-key prediction security of $F \in\{$ GKS, OKS, FKS $\}$ against an adversary $\mathcal{A}$ is defined as

$$
\begin{array}{r}
\operatorname{Adv}_{F}^{u \text {-key-pre }}(\mathcal{A})=\operatorname{Pr}\left(\pi \stackrel{\$}{\leftarrow} \operatorname{perm}(b), \mathcal{Q} \leftarrow \mathcal{A}^{\pi^{ \pm}}, K_{1}, \ldots, K_{u} \stackrel{\$}{\leftarrow}\{0,1\}^{k}:\right. \\
\left.\exists i \in\{1, \ldots, u\} \text { such that } K_{i} \in \operatorname{yield}_{c_{\mathrm{ab}}, \lambda}(\mathcal{Q})\right) .
\end{array}
$$

For $N \geq 0$, we define by $\operatorname{Adv}_{F}^{u \text {-key-pre }}(N)$ the maximum over all adversaries making $N$ queries to its oracle.

Theorem 1 and the proof likewise generalize straightforwardly.

Theorem 2. Let $u \in \mathbb{N}$. Consider $F=$ OKS for parameters $(b, c, r, k)$, where $\lambda:=$ $\lceil k / r\rceil>1$. Let $\alpha>\lambda$ be a natural number. Let $0 \leq N \leq 2^{b-1}$. We have

$$
\operatorname{Adv}_{F}^{u \text {-key-pre }}(N) \leq u \cdot \frac{(2 \alpha)^{\lambda-1} N}{2^{k}}+\lambda 2^{\lambda} 2^{2 c}\left(\frac{2 e N^{*}}{2^{c} \alpha}\right)^{\alpha / \lambda}
$$

where $N^{*}=\max \left\{N, \lambda 2^{(\lambda-1) r}\right\}$.

Proof. The core of the proof of Theorem 1 is about deriving an upper bound on $\left|\operatorname{yield}_{c, \lambda}(\mathcal{Q})\right|$. Once this bound is derived - the derivation of Section 3.4 carries over verbatim - any of the $u$ keys is in the yield with probability at most $u \cdot\left|\operatorname{yield}_{c, \lambda}(\mathcal{Q})\right| / 2^{k} \leq u \cdot \frac{(2 \alpha)^{\lambda-1} N}{2^{k}}$.

\section{Application to Keyed Sponge and Duplex}

Keyed sponges are evaluated in a PRF security model. In more detail, let $\mathcal{R O}^{\infty}:\{0,1\}^{*} \rightarrow$ $\{0,1\}^{\infty}$ be a function that for every input $m$ defines an infinitely large string of random bits, and define $\mathcal{R O}:\{0,1\}^{*} \times \mathbb{N} \rightarrow\{0,1\}^{\mathbb{N}}$ as a function that on input of $(m, \ell) \in\{0,1\}^{*} \times \mathbb{N}$ outputs $\left\lceil\mathcal{R} \mathcal{O}^{\infty}(m)\right\rceil_{\ell}$. Abusing notation, write $\operatorname{ro}(*, \mathbb{N})$ for the set of all such functions $\mathcal{R O}$. 
For a random permutation $\pi \stackrel{\$}{\leftarrow}$ perm $(b)$, key $K \stackrel{\$}{\leftarrow}\{0,1\}^{k}$, and $\mathcal{R O} \stackrel{\$}{\leftarrow} \operatorname{ro}(*, \mathbb{N})$, consider an adversary $\mathcal{A}$ that has oracle access to either $\left(F_{K}^{\pi}, \pi^{ \pm}\right)$or $\left(\mathcal{R O}, \pi^{ \pm}\right)$. It succeeds if it manages to determine (with high probability) the world it is conversing with. Formally:

Definition 5. The PRF security of $F \in\{\mathrm{GKS}, \mathrm{OKS}, \mathrm{FKS}\}$ against an adversary $\mathcal{A}$ is defined as

$$
\begin{aligned}
\operatorname{Adv}_{F}^{\operatorname{prf}}(\mathcal{A})=\operatorname{Pr}\left(\pi \stackrel{\$}{\leftarrow} \operatorname{perm}(b), K \stackrel{\$}{\leftarrow}\{0,1\}^{k}: 1 \leftarrow \mathcal{A}^{F_{K}^{\pi}, \pi^{ \pm}}\right) & \\
& \quad \operatorname{Pr}\left(\pi \stackrel{\$}{\leftarrow} \operatorname{perm}(b), \mathcal{R O} \stackrel{\$}{\leftarrow} \operatorname{ro}(*, \mathbb{N}): 1 \leftarrow \mathcal{A}^{\mathcal{R O}, \pi^{ \pm}}\right) .
\end{aligned}
$$

For $M, N \geq 0$, we define by $\mathbf{A d v}_{F}^{\operatorname{prf}}(M, N)$ the maximum over all adversaries with query complexity $M$ to the first oracle (in terms of the number of underlying primitive calls) and $N$ to the second oracle.

A bound on the PRF security of FKS was derived by Mennink et al. [MRV15], but we are mostly concerned with the outer keyed sponge. For OKS, an earlier proof appeared by Bertoni et al. [BDPV11c]. Andreeva et al. [ADMV15] improved the analysis and generalized it to multi-key security. Naito and Yasuda [NY16] derived a bound that is independent of the message length. The improvements in these bounds come at the cost of defining extra security parameters, such as the multiplicity, the number of oracles, the number of construction queries, and the maximum length of each construction query. As our security model considers an adversary whose complexity is solely measured by $M$ and $N$, we discard most of the sophisticated improvements in [ADMV15, NY16] and consider a simplified bound. In addition, we modernize the bound using the key prediction security notion of Definition 3.

Theorem 3 (Andreeva et al. [ADMV15] and Naito and Yasuda [NY16], simplified). Consider $F=\mathrm{OKS}$ for parameters $(b, c, r, k)$. Let $M, N \geq 0$. We have

$$
\mathbf{A d v}_{F}^{\mathrm{prf}}(M, N) \leq \text { const }_{1} \cdot \frac{M^{2}}{2^{c}}+\text { const }_{2} \cdot \frac{M N}{2^{c}}+\mathbf{A d v}_{F}^{\text {key-pre }}(N) .
$$

In $[\mathrm{ADMV} 15]$, the constant terms satisfy const $_{1}=1$ and const $_{2} \leq 4$. Also for the bound of [NY16] the constant terms are reasonably small, but as their analysis is more involved, a neat approximation for these terms cannot be easily derived. The simplifications we have put through from [ADMV15, NY16] only affect the fractions in (28) and do not affect the point we are making with regard to the remaining term in (28). This term $\mathbf{A d v}_{F}^{\text {key-pre }}(N)$ in turn corresponds to a specific bad event in the analyses in [ADMV15, NY16]: assuming that the adversary never makes the primitive queries related to the absorption of the key, the state after the absorption of the key has a sufficient amount of entropy. Both Andreeva et al. [ADMV15, Lemma 2] and Naito and Yasuda [NY16, Theorem 2] rely on the bound of Gaži et al. [GPT15a, GPT15b] on $\mathbf{A d v}_{F}^{\text {key-pre }}(N)$ : the bound expressed in Proposition 1. Our new result of Theorem 1 directly improves over the bounds from Andreeva et al. and Naito and Yasuda, and confirms that a shorter key can be taken to achieve the same level of security.

On passing, we remark that the result has comparable impact to the keyed duplex [BDPV11a], a sponge-related construction well-suited for authenticated encryption. It is a stateful construction that has a "duplexing interface:" it gets as input a data block of size $r$ bits, transforms the state using a permutation, and returns part of the outer part of the state. It comes with an outer-keyed flavor [BDPV11a] as well as a full-keyed flavor [MRV15, DMV17], and the keyed sponge bounds are known to be transferable to the duplex (and vice versa) up to some degree. Crucial to this transition is that in the keyed duplex, the key absorption occurs with no intermediate output of the outer part, and thus 
occurs in a full-/outer-keyed sponge fashion. In this way, the key prediction term in the duplex bounds is also $\mathbf{A} \mathbf{d} \mathbf{v}_{F}^{\text {key-pre }}(N)$, where $F \in\{\mathrm{OKS}, \mathrm{FKS}\}$, i.e., the bound derived in Theorem 1.

Finally, the generic security results on HMAC-SHA-3 [NW16] and the sandwich sponge [Nai16] rely on the key prediction security term of Gaži et al. in the exact same way, and our new bound immediately improves their results.

\section{Note on Key Recovery}

In a similar vein as in Section 3, one can define the key recovery security of $F \in$ $\{\mathrm{GKS}, \mathrm{OKS}, \mathrm{FKS}\}$. For a random permutation $\pi \stackrel{\$}{\leftarrow} \operatorname{perm}(b)$ and key $K \stackrel{\$}{\leftarrow}\{0,1\}^{k}$, consider an adversary $\mathcal{A}$ that has oracle access to $\left(F_{K}^{\pi}, \pi^{ \pm}\right)$. The adversary can make a limited amount of queries to its oracles, summarized in a transcript $\mathcal{Q}$, and afterwards it outputs a key $K^{\prime} \in\{0,1\}^{k}$. It wins if $K^{\prime} \in \operatorname{yield}_{c_{\mathrm{ab}}, \lambda}(\mathcal{Q})$ and $F_{K}^{\pi}(\cdot)=F_{K^{\prime}}^{\pi}(\cdot)$. Formally:

Definition 6. The key recovery security of $F \in\{$ GKS, OKS, FKS $\}$ against an adversary $\mathcal{A}$ is defined as

$$
\begin{aligned}
\operatorname{Adv}_{F}^{\text {key-rec }}(\mathcal{A})=\operatorname{Pr}\left(\pi \stackrel{\$}{\leftarrow} \operatorname{perm}(b), K \stackrel{\$}{\leftarrow}\{0,1\}^{k},\left(K^{\prime}, \mathcal{Q}\right) \leftarrow \mathcal{A}_{K}^{F^{\pi}, \pi^{ \pm}}:\right. \\
\left.K^{\prime} \in \operatorname{yield}_{c_{\mathrm{ab}, \lambda}}(\mathcal{Q}) \wedge F_{K}^{\pi}(\cdot)=F_{K^{\prime}}^{\pi}(\cdot)\right) .
\end{aligned}
$$

For $M, N \geq 0$, we define by $\operatorname{Adv}_{F}^{\text {key-rec }}(M, N)$ the maximum over all adversaries with query complexity $M$ to the first oracle (in terms of the number of underlying primitive calls) and $N$ to the second oracle.

In other words, key recovery security differs from key prediction security in that the adversary has access to the keyed construction $F_{K}^{\pi}$.

One may argue that key recovery is a more meaningful notion to consider than key prediction. However, close inspection at how a key recovery security proof would look like reveals that the key recovery security of OKS is very close to its PRF security. To wit, the core ingredients of the PRF security bound of OKS (Theorem 3) are (i) the event of two evaluations of $\pi$ imposed by $F_{K}^{ \pm}$with the same inner part, (ii) a primitive query to $\pi^{ \pm}$and an evaluation of $\pi$ imposed by $F_{K}^{ \pm}$with the same inner part, and (iii) guessing/predicting the key. These parts are represented by the three terms in the bound of Theorem 3 in equal order.

Obviously, one way to recover the key is to predict it (part (iii) of the above). Now, suppose the adversary makes a query to $\pi^{ \pm}$whose inner part is equal to the inner part of an evaluation of $\pi$ imposed by $F_{K}^{ \pm}$(part (ii) of the above). In this case, the adversary can back-track the sponge to obtain $t_{\lambda}$, the state of the sponge after the absorption of the last key block. Once it knows $t_{\lambda}$, depending on $\lambda$ it can learn (part of) the key. Regarding part (i): if two evaluations of $\pi$ imposed by $F_{K}^{ \pm}$have the same inner part, the adversary can use this information to distinguish the scheme from a random function but it has no means to use this information to recover the key.

To summarize, the key recovery security bound would be constituted of parts (iii) and (ii) of the PRF security bound. Part (i) is minor compared with (ii), as the offline complexity is typically higher than the online complexity. We can thus conclude that the key recovery security of OKS is very close to its PRF security. 


\section{Acknowledgments}

Bart Mennink is supported by a postdoctoral fellowship from the Netherlands Organisation for Scientific Research (NWO) under Veni grant 016. Veni.173.017. The author would like to thank the reviewers for their detailed comments and suggestions.

\section{References}

$\left[\mathrm{ABB}^{+} 14\right]$ Elena Andreeva, Begül Bilgin, Andrey Bogdanov, Atul Luykx, Florian Mendel, Bart Mennink, Nicky Mouha, Qingju Wang, and Kan Yasuda. PRIMATEs v1.02, September 2014.

[ADMV15] Elena Andreeva, Joan Daemen, Bart Mennink, and Gilles Van Assche. Security of Keyed Sponge Constructions Using a Modular Proof Approach. In Gregor Leander, editor, FSE 2015, volume 9054 of Lecture Notes in Computer Science, pages 364-384. Springer, 2015.

[AHMN10] Jean-Philippe Aumasson, Luca Henzen, Willi Meier, and María Naya-Plasencia. Quark: A Lightweight Hash. In Stefan Mangard and François-Xavier Standaert, editors, CHES 2010, volume 6225 of Lecture Notes in Computer Science, pages 1-15. Springer, 2010.

[AJN16] Jean-Philippe Aumasson, Philipp Jovanovic, and Samuel Neves. NORX v3.0, September 2016.

$\left[\mathrm{BDP}^{+}\right.$16a] Guido Bertoni, Joan Daemen, Michaël Peeters, Gilles Van Assche, and Ronny Van Keer. CAESAR submission: Ketje v2, September 2016.

$\left[\mathrm{BDP}^{+} 16 \mathrm{~b}\right]$ Guido Bertoni, Joan Daemen, Michaël Peeters, Gilles Van Assche, and Ronny Van Keer. CAESAR submission: Keyak v2, September 2016.

[BDPV07] Guido Bertoni, Joan Daemen, Michaël Peeters, and Gilles Van Assche. Sponge functions. ECRYPT Hash Workshop 2007, May 2007.

[BDPV08] Guido Bertoni, Joan Daemen, Michaël Peeters, and Gilles Van Assche. On the Indifferentiability of the Sponge Construction. In Nigel P. Smart, editor, EUROCRYPT 2008, volume 4965 of Lecture Notes in Computer Science, pages 181-197. Springer, 2008.

[BDPV11a] Guido Bertoni, Joan Daemen, Michaël Peeters, and Gilles Van Assche. Duplexing the Sponge: Single-Pass Authenticated Encryption and Other Applications. In Ali Miri and Serge Vaudenay, editors, SAC 2011, volume 7118 of Lecture Notes in Computer Science, pages 320-337. Springer, 2011.

[BDPV11b] Guido Bertoni, Joan Daemen, Michaël Peeters, and Gilles Van Assche. The Keccak reference, January 2011. https://keccak.team/files/ Keccak-reference-3.0.pdf.

[BDPV11c] Guido Bertoni, Joan Daemen, Michaël Peeters, and Gilles Van Assche. On the security of the keyed sponge construction. Symmetric Key Encryption Workshop, February 2011.

[BDPV12] Guido Bertoni, Joan Daemen, Michaël Peeters, and Gilles Van Assche. Permutation-based encryption, authentication and authenticated encryption. Directions in Authenticated Ciphers, July 2012. 
$\left[\mathrm{BKL}^{+} 11\right]$ Andrey Bogdanov, Miroslav Knezevic, Gregor Leander, Deniz Toz, Kerem Varıcı, and Ingrid Verbauwhede. spongent: A Lightweight Hash Function. In Bart Preneel and Tsuyoshi Takagi, editors, CHES 2011, volume 6917 of Lecture Notes in Computer Science, pages 312-325. Springer, 2011.

$\left[\mathrm{BKL}^{+} 17\right]$ Daniel J. Bernstein, Stefan Kölbl, Stefan Lucks, Pedro Maat Costa Massolino, Florian Mendel, Kashif Nawaz, Tobias Schneider, Peter Schwabe, FrançoisXavier Standaert, Yosuke Todo, and Benoît Viguier. Gimli : A Cross-Platform Permutation. In Wieland Fischer and Naofumi Homma, editors, CHES 2017, volume 10529 of Lecture Notes in Computer Science, pages 299-320. Springer, 2017.

[CAE18] CAESAR: Competition for Authenticated Encryption: Security, Applicability, and Robustness, January 2018. http://competitions.cr.yp.to/caesar. html.

$\left[\mathrm{CDH}^{+}{ }^{12}\right]$ Donghoon Chang, Morris Dworkin, Seokhie Hong, John Kelsey, and Mridul Nandi. A keyed sponge construction with pseudorandomness in the standard model. NIST SHA-3 Workshop, March 2012.

[CG16] Colin Chaigneau and Henri Gilbert. Is AEZ v4.1 Sufficiently Resilient Against Key-Recovery Attacks? IACR Trans. Symmetric Cryptol., 2016(1):114-133, 2016.

[DEMS16] Christoph Dobraunig, Maria Eichlseder, Florian Mendel, and Martin Schläffer. Ascon v1.2, September 2016. Submission to CAESAR competition.

[DMV17] Joan Daemen, Bart Mennink, and Gilles Van Assche. Full-State Keyed Duplex with Built-In Multi-user Support. In Tsuyoshi Takagi and Thomas Peyrin, editors, ASIACRYPT 2017, Part II, volume 10625 of Lecture Notes in Computer Science, pages 606-637. Springer, 2017.

[FFL12] Ewan Fleischmann, Christian Forler, and Stefan Lucks. McOE: A Family of Almost Foolproof On-Line Authenticated Encryption Schemes. In Anne Canteaut, editor, FSE 2012, volume 7549 of Lecture Notes in Computer Science, pages 196-215. Springer, 2012.

[FIP99] FIPS 197: Advanced Encryption Standard (AES). National Institute of Standards and Technology, 1999.

[FIP15] FIPS 202: SHA-3 Standard: Permutation-Based Hash and Extendable-Output Functions, 2015.

[FLS15] Thomas Fuhr, Gaëtan Leurent, and Valentin Suder. Collision Attacks Against CAESAR Candidates - Forgery and Key-Recovery Against AEZ and Marble. In Iwata and Cheon [IC15], pages 510-532.

[GPP11] Jian Guo, Thomas Peyrin, and Axel Poschmann. The PHOTON Family of Lightweight Hash Functions. In Phillip Rogaway, editor, CRYPTO 2011, volume 6841 of Lecture Notes in Computer Science, pages 222-239. Springer, 2011.

[GPT15a] Peter Gaži, Krzysztof Pietrzak, and Stefano Tessaro. The Exact PRF Security of Truncation: Tight Bounds for Keyed Sponges and Truncated CBC. In Rosario Gennaro and Matthew Robshaw, editors, CRYPTO 2015, Part I, volume 9215 of Lecture Notes in Computer Science, pages 368-387. Springer, 2015 . 
[GPT15b] Peter Gaži, Krzysztof Pietrzak, and Stefano Tessaro. Tight Bounds for Keyed Sponges and Truncated CBC. Cryptology ePrint Archive, Report 2015/053, 2015.

[Guo14] Jian Guo. Marble Specification Version 1.0, March 2014.

[Ham17a] Michael Hamburg. STROBE protocol framework, July 2017. https://strobe. sourceforge.io.

[Ham17b] Mike Hamburg. The STROBE protocol framework. Cryptology ePrint Archive, Report 2017/003, 2017.

[HKR14] Viet Tung Hoang, Ted Krovetz, and Phillip Rogaway. AEZ v3: Authenticated Encryption by Enciphering, August 2014.

[HKR15a] Viet Tung Hoang, Ted Krovetz, and Phillip Rogaway. AEZ v4: Authenticated Encryption by Enciphering, August 2015.

[HKR15b] Viet Tung Hoang, Ted Krovetz, and Phillip Rogaway. AEZ v4.1: Authenticated Encryption by Enciphering, October 2015.

[IC15] Tetsu Iwata and Jung Hee Cheon, editors. ASIACRYPT 2015, Part II, volume 9453 of Lecture Notes in Computer Science. Springer, 2015.

[JLM14] Philipp Jovanovic, Atul Luykx, and Bart Mennink. Beyond $2^{c / 2}$ Security in Sponge-Based Authenticated Encryption Modes. In Palash Sarkar and Tetsu Iwata, editors, ASIACRYPT 2014, Part I, volume 8873 of Lecture Notes in Computer Science, pages 85-104. Springer, 2014.

$\left[\mathrm{KLL}^{+}{ }^{14}\right]$ Elif Bilge Kavun, Martin M. Lauridsen, Gregor Leander, Christian Rechberger, Peter Schwabe, and Tolga Yalçın. Prøst v1.1, June 2014.

[MMRT12] Florian Mendel, Bart Mennink, Vincent Rijmen, and Elmar Tischhauser. A Simple Key-Recovery Attack on McOE-X. In Josef Pieprzyk, Ahmad-Reza Sadeghi, and Mark Manulis, editors, CANS 2012, volume 7712, pages 23-31. Springer, 2012.

[MRH04] Ueli M. Maurer, Renato Renner, and Clemens Holenstein. Indifferentiability, Impossibility Results on Reductions, and Applications to the Random Oracle Methodology. In Moni Naor, editor, TCC 2004, volume 2951 of Lecture Notes in Computer Science, pages 21-39. Springer, 2004.

[MRV15] Bart Mennink, Reza Reyhanitabar, and Damian Vizár. Security of Full-State Keyed Sponge and Duplex: Applications to Authenticated Encryption. In Iwata and Cheon [IC15], pages 465-489.

[Nai16] Yusuke Naito. Sandwich Construction for Keyed Sponges: Independence Between Capacity and Online Queries. In Sara Foresti and Giuseppe Persiano, editors, CANS 2016, volume 10052 of Lecture Notes in Computer Science, pages 245-261, 2016.

[NIS16] NIST Special Publication 800-185: SHA-3 Derived Functions: cSHAKE, KMAC, TupleHash and ParallelHash, 2016.

[NW16] Yusuke Naito and Lei Wang. Replacing SHA-2 with SHA-3 Enhances Generic Security of HMAC. In Kazue Sako, editor, CT-RSA 2016, volume 9610 of Lecture Notes in Computer Science, pages 397-412. Springer, 2016. 
[NY16] Yusuke Naito and Kan Yasuda. New Bounds for Keyed Sponges with Extendable Output: Independence Between Capacity and Message Length. In Thomas Peyrin, editor, FSE 2016, volume 9783 of Lecture Notes in Computer Science, pages 3-22. Springer, 2016. 